\title{
Atividade eletromiográfica durante o agachamento unipodal associado a diferentes posições do pé
}

\author{
Gabriel Ribeiroㅁ, Valdeci Carlos Dionísioํㅜ e Gil Lúcio Almeida ${ }^{1}$
}

\section{RESUMO}

O objetivo principal deste estudo foi quantificar a atividade muscular durante a realização de agachamento unipodal com variações na técnica. Oito voluntários saudáveis realizaram agachamentos associados a cinco tipos de posição do pé: posição neutra, sobre cunha com $10^{\circ}$ de declive, sobre cunha com $10^{\circ}$ de aclive, sobre cunha com $10^{\circ}$ de inclinação medial e sobre cunha com $10^{\circ}$ de inclinação lateral. Foram avaliados os dados eletromiográficos dos músculos vasto medial oblíquo, vasto lateral, reto femoral, bíceps femoral, gastrocnêmio lateral e tibial anterior, utilizando a ANOVA fator único. O valor eletromiográfico integrado de todos os músculos não foi estatisticamente diferente nos cinco tipos de posição do pé. Os resultados deste estudo sugerem que diferentes tipos de posicionamento do pé durante o agachamento unipodal não provocam alterações no padrão de recrutamento muscular.

\section{ABSTRACT \\ Electromyographic activity during one-legged squatting under different foot positions}

The specific aim of this study was to quantify muscle activity while performing squat with technique variations. Eight healthy volunteers performed squats under 5 types of foot positions: neutral position, under $10^{\circ}$ descending wedge, under a $10^{\circ}$ ascending wedge, under a $10^{\circ}$ medial wedge and under a $10^{\circ}$ lateral wedge. It was evaluated electromyographic data of vastus medialis oblique, vastus lateralis, rectus femoris, biceps femoris, lateral gastrocnemius and tibialis anterior muscles, using One-Way ANOVA. The EMG integrated values was not significantly different across the 5 foot positions. The results of this study suggest that different foot positions during one-legged squat do not cause changes in muscle recruitment.

\section{RESUMEN}

\section{Actividad electromiográfica durante el agache unipodal asociado a diferentes posiciones del pie}

El objetivo principal de este estudio ha sido cuantificar la actividad muscular durante la realización de agachamiento unipodal con variaciones en la técnica. Ocho voluntarios saludables realizaron agachamientos asociados a cinco tipos de posición del pie: posición neutra, sobre cuña con $10^{\circ}$ de declive, sobre cuña con $10^{\circ}$ de elevación, sobre cuña con $10^{\circ}$ de inclinación media y sobre cuña con $10^{\circ}$ de inclinación lateral. Fueron evaluados los datos electromiográficos de los músculos vasto medial oblicuo, vasto lateral,

1. Laboratório de Estudos Clínicos em Fisioterapia, Universidade de Ribeirão Preto, Ribeirão Preto.

Recebido em 21/6/05. Versão final recebida em 7/12/05. Aceito em 17/7/06. Endereço para correspondência: Avenida do Café, 1.139, Vila Amélia 14050-230 - Ribeirão Preto, SP. Tels.: (16) 3966-5406/(16) 602-3079. Email: fta_gabrielribeiro@yahoo.com.br

\author{
Palavras-chave: Músculo. Descarga de peso. Reabilitação. J oelho. \\ Keywords: $\quad$ Muscle. Weight-Bearing. Rehabilitation. Knee. \\ Palabras-clave: Músculo. Descarga de peso. Rehabilitación. Rodilla.
}

recto femoral, bíceps femoral, gastrocnemio lateral y tibial anterior, utilizando ANOVA factor único. El valor electromiográfico integrado de todos los músculos no fue estadísticamente diferente en los cinco tipos de posición del pie. Los resultados de este estudio sugieren que diferentes tipos de posicionamiento del pie durante el proceso de agachamiento unipodal no provocan alteraciones en el padrón de reclutamiento muscular.

\section{INTRODUÇÃO}

O exercício de agachamento tem sido muito utilizado nos últimos anos nos programas de treinamento e de reabilitação do membro inferior. O emprego de variações desse exercício, com o intuito de observar diferentes padrões de atividade muscular, tem sido muito freqüente ${ }^{(1-7)}$. O conhecimento do padrão de atividade muscular durante o agachamento pode aumentar a seletividade de programas de prevenção, reabilitação e treinamento esportivo do membro inferior(8).

A utilização de órteses ${ }^{(6)}$, diferentes distâncias ${ }^{(2,4,7)}$ e posições dos pés ${ }^{(3)}$ e o emprego de diferentes graus de rotação da extremidade inferior ${ }^{(1,4-5)}$ foram algumas das estratégias utilizadas para tentar desencadear um recrutamento muscular seletivo durante o exercício de agachamento.

Durante o agachamento unipodal, o comportamento eletromiográfico da musculatura do quadríceps foi estudado associado a diferentes posicionamentos do pé no sentido médio-lateral (3). Neste estudo, não foram observadas diferenças no comportamento eletromiográfico (EMG) de dois músculos uniarticulares(3), vasto medial oblíquo e vasto lateral. Por outro lado, Hertel et al.(6) encontraram diferenças no padrão de atividade EMG nesta mesma tarefa utilizando uma órtese para correção da pronação e supinação do pé. Entretanto, o posicionamento do tronco e a velocidade angular do joelho não foram controlados em nenhum destes estudos, o que poderia influenciar o padrão de recrutamento muscular (9-12).

Hipotetizamos que com o controle da velocidade angular do joeIho e do deslocamento do tronco no plano sagital e frontal, podeníamos observar um padrão de atividade muscular diferente, durante o agachamento unipodal associado a diferentes inclinações do pé no sentido médio-lateral e ântero-posterior.

Como a tarefa de agachamento unipodal é empregada freqüentemente em atividades de reabilitação(13-14) e em testes utilizados nos exames físicos pré-participação(15-16), este estudo pode contribuir para a compreensão desta tarefa, além de ajudar na elaboração de programas de reabilitação.

Dessa forma, o objetivo deste estudo foi quantificar a atividade muscular durante a realização de agachamento unipodal com variações na técnica e com controle de dois parâmetros cinemáticos - velocidade angular do joelho e deslocamento de tronco. 


\section{METODOLOGIA}

\section{Sujeitos}

Oito sujeitos saudáveis (quatro homens, quatro mulheres) e destros, com idade entre 18 e 24 anos, participaram deste estudo. O histórico médico de todos os sujeitos foi avaliado; foram selecionados os sujeitos que não apresentaram qualquer deficiência orgânica, cirurgia ou dor na coluna vertebral e nos membros inferiores. A estatística descritiva destes sujeitos encontra-se na tabela 1.

\begin{tabular}{lccc}
\hline & \multicolumn{2}{c}{ TABELA 1 } \\
& $\begin{array}{c}\text { Estatística descritiva para homens }(\mathbf{n}=\mathbf{4}), \\
\text { mulheres }(\mathbf{n}=\mathbf{4}) \text { e amostra total }(\mathbf{n}=\mathbf{8}) *\end{array}$ \\
\hline Idade (anos) & Altura (cm) & Peso (kg) \\
Homens & $19,7 \pm 1,0$ & $1,7 \pm 0,0$ & $69,6 \pm 0,9$ \\
Mulheres & $22,7 \pm 0,7$ & $1,6 \pm 0,0$ & $54,7 \pm 1,8$ \\
Amostra total & $21,2 \pm 0,8$ & $1,6 \pm 0,0$ & $62,1 \pm 2,97$ \\
\hline
\end{tabular}

* Valores expressos como média \pm EPM.

Para participação no experimento, antes da coleta de dados, todos os sujeitos leram e assinaram um consentimento formal aprovado pelo Comitê de Ética em Pesquisa da Universidade de Ribeirão Preto.

\section{Instrumentação}

\section{Registro dos dados eletromiográficos (EMG)}

Para a coleta dos dados, o local de cada eletrodo foi depilado e limpo com álcool para facilitar aderência do eletrodo e a condução de sinais de EMG. Eletrodos de superfície bipolar $(10 \times 1 \mathrm{~mm})$ DelSYS (modelo DE2.2L) foram posicionados com uma distância de $3 \mathrm{~cm}$ entre os eletrodos. Para fixação dos eletrodos no músculo vasto medial oblíquo (VMO) foram seguidos os procedimentos descritos por Bevilaqua-Grossi et al.(17). Para os músculos reto femoral (RF), vasto lateral (VL), bíceps femoral (BF), gastrocnêmio lateral $(G L)$ e tibial anterior (TA), os eletrodos foram fixados de acordo com os procedimentos empregados por Basmajian e Blumenstein $^{(18)}$. Em todas coletas o local foi identificado e preparado pelo mesmo pesquisador. Os sinais de EMG foram ampliados ( $x 2000$ ), filtrados (band pass $20-450 \mathrm{~Hz}$ ) e registrados. Os dados foram digitalizados a 12 bits, coletados por um computador da IBM-PC a $1.000 \mathrm{~Hz}$ e expressos em milivolts $(\mathrm{mV})$. Os sinais de EMG não foram normalizados, pois as comparações são feitas entre o mesmo músculo e não entre músculos diferentes.

\section{Registro dos dados cinemáticos}

Para a análise do movimento, foram registradas as coordenadas $X, Y$ e $Z$ utilizando LEDs (light emitting diode) que foram fixados no centro das articulações do ombro, quadril, joelho e tornozelo (extremidade lateral do acrômio, trocanter maior do fêmur, epicôndilo lateral do fêmur e maléolo lateral) (figura 1). A emissão do sinal infravermelho destas marcas foi capturada com freqüência de $100 \mathrm{~Hz}$ por um sistema óptico tridimensional Optotrak 3020.

\section{Procedimento}

\section{Agachamento unipodal com o pé na posição neutra (PN)}

Cada sujeito foi posicionado em pé, com o membro direito no centro de um quadrado $(80 \times 80 \mathrm{~cm})$ desenhado no chão. Foi solicitado que cada participante mantivesse o joelho esquerdo flexionado aproximadamente $60^{\circ}$. O sujeito manteve o joelho direito em extensão total e os membros superiores estendidos e elevados à frente com um ângulo de $90^{\circ}$ de flexão de ombro. Um painel de vidro foi colocado à frente do sujeito com marcas determinando os alvos. Essas marcas delimitavam a posição inicial e a posição final do movimento e representavam uma amplitude de $35^{\circ}$ a $40^{\circ}$ de flexão de joelho, tendo como referência o deslocamento do braço. A partir da posição inicial, ao receber um comando verbal, o sujeito realizou o agachamento unipodal por meio da flexão do joelho. Os sujeitos foram motivados a realizar cada movimento o mais rápido possível. Ao chegar ao alvo o sujeito mantinha-se na posição até receber outro comando verbal para voltar à posição inicial. Apenas a fase descendente do agachamento foi analisada. Foi realizada uma série de 10 movimentos de agachamento. A figura 1 representa de forma esquemática o agachamento unipodal com o pé na posição neutra.

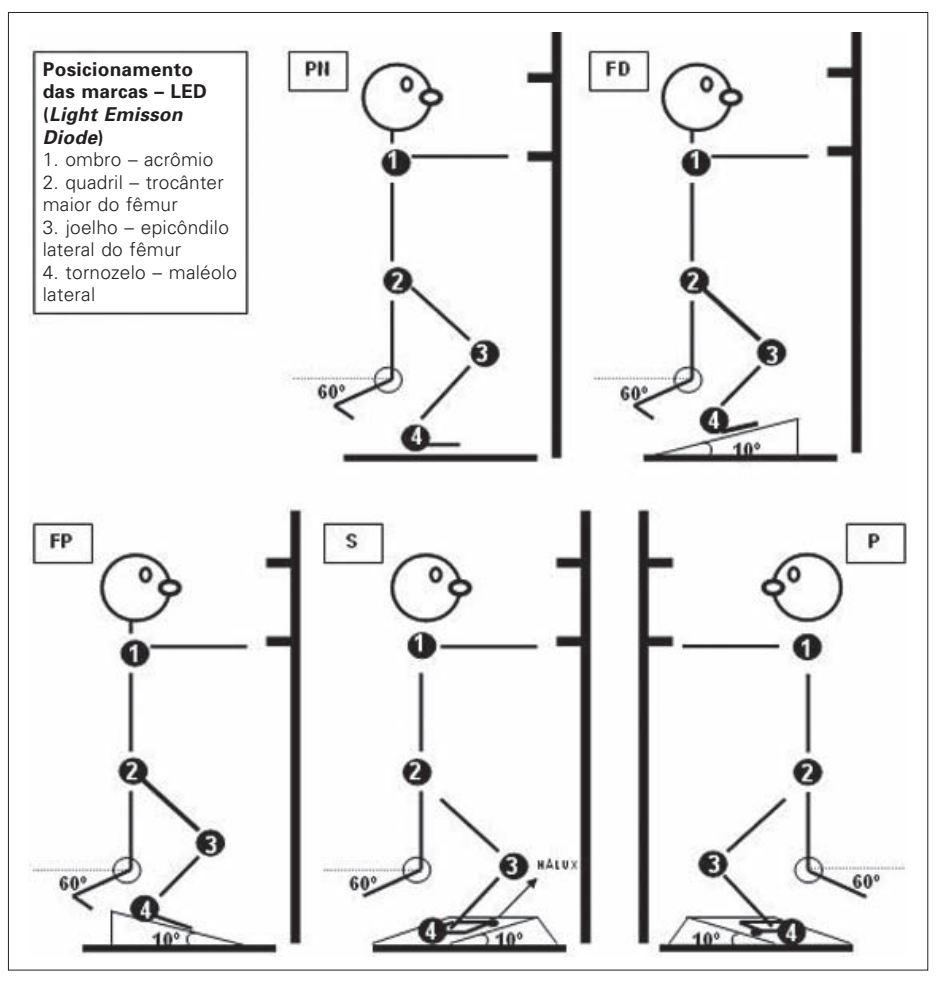

Figura 1 - Representação esquemática do posicionamento dos indivíduos após realização do agachamento unipodal. $P N=$ posição neutra; $F D=$ flexão dorsal; FP = flexão plantar; $S=$ supinação; $P=$ pronação.

Agachamentos unipodais com o pé nas posições de flexão plan$\operatorname{tar}(F P)$, flexão dorsal (FD), supinação $(S)$ e pronação $(P)$

Para a realização dos agachamentos com o pé direito nas posições de FP, FD, S, e P foi realizado o mesmo procedimento descrito acima, associado à utilização de uma cunha $(55 \times 55 \mathrm{~cm})$ de madeira. Esta cunha com $10^{\circ}$ de inclinação foi direcionada de forma a posicionar o pé em $10^{\circ}$ de $\mathrm{FP}, 10^{\circ}$ de $\mathrm{FD}, 10^{\circ}$ de $\mathrm{S}$ e $10^{\circ}$ de $\mathrm{P}$ antes do início do agachamento. A escolha de uma cunha com $10^{\circ}$ de inclinação foi baseada no estudo de Hung e Gross $^{(3)}$. A figura 1 representa de forma esquemática o agachamento unipodal com o pé posicionado em flexão plantar.

\section{Processamento dos dados}

Os sinais eletromiográficos (EMG) e os registros das marcas $X$, Y e Z foram sincronizados por um sincronizador ODAU I/ (Optotrak Dates Acquisition Unit II), e depois tratados matematicamente por um código em MatLab (Math Works Inc., versão 6.0). Neste código foi calculado o deslocamento linear das marcas, o deslocamento e velocidade angular do joelho, e quantificados os sinais EMG de cada músculo. Todas as tarefas de agachamento que tiveram um deslocamento linear maior que três centímetros, nos sentidos ântero-posterior e látero-lateral, foram descartadas com o intuito reduzir as interferências provenientes do equilíbrio da cada sujeito. O deslocamento linear da marca posicionada no acrômio foi utilizado como referência para a realização deste procedimento.

Os sinais eletromiográficos foram calculados na fase descendente do agachamento, utilizando-se como referência para o cál- 
culo a velocidade angular do joelho. O início do movimento foi considerado quando a velocidade inicial alcançou $5 \%$ do pico da velocidade e o término do movimento quando a velocidade final alcançou $5 \%$ do pico da velocidade. Durante o movimento foram consideradas duas fases (aceleração e desaceleração). A fase de aceleração foi considerada entre o início do movimento e o pico da velocidade e a fase de desaceleração foi considerada entre o pico da velocidade e o final do movimento.

A velocidade do joelho foi utilizada para quantificar os sinais eletromiográficos dos músculos vasto medial oblíquo (VMO), reto femoral (RF), vasto lateral (VL), bíceps femoral (BF), gastrocnêmio lateral (GL) e tibial anterior (TA). Os sinais eletromiográficos (EMG) foram integrados na fase de aceleração e na fase de desaceleração do movimento. Para cada sujeito, a média da EMG de cada músculo foi calculada para cada tipo de agachamento.

\section{Análise dos dados}

Todos os dados utilizados na análise estatística foram submetidos ao teste de Kolmogorov-Smirnov, para verificar se os mesmos apresentavam uma distribuição normal. Todos os dados analisados apresentaram uma distribuição normal. Para testar o efeito do tipo de agachamento sobre o sinal eletromiográfico de cada músculo individualmente foi utilizada a análise de variância (ANOVA) para fator único. A mesma análise foi empregada para comparar deslocamento, velocidade angular do joelho e o deslocamento do tronco entre os diferentes tipos de agachamento. Em todas as avaliações o nível de significância considerado foi inferior ou igual a $5 \%(p \leq 0,05)$.

\section{RESULTADOS}

\section{Deslocamento e velocidade angular}

A amplitude do deslocamento angular do joelho e a velocidade angular da articulação do joelho não foram estatisticamente diferentes (tabela 2) entre os cinco tipos de agachamento realizados com diferentes posições do pé (PN, FP, FD, S e P). Os valores do deslocamento angular estão expressos na figura 2.

\section{Análise eletromiográfica}

A análise estatística não demonstrou efeito dos diferentes tipos de agachamento unipodal sobre a atividade eletromiográfica
(EMG) dos músculos analisados nas fases de aceleração e desaceleração (tabela 3). A EMG dos músculos analisados nas fases de aceleração e desaceleração do movimento de agachamento unipodal está representada na figura 3.

TABELA 2

Análise de variância (ANOVA) - Parâmetros cinemáticos

\begin{tabular}{lccc}
\hline & F & Graus de liberdade & p \\
Deslocamento angular do joelho & 1,27 & $(4,28)$ & $<0,30$ \\
Velocidade angular do joelho & 0,85 & $(4,28)$ & $<0,50$ \\
\hline
\end{tabular}
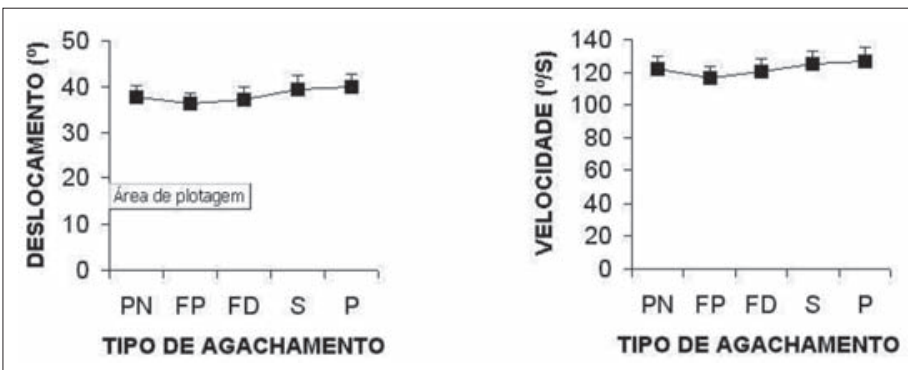

Figura 2 - Deslocamento e velocidade angular da articulação do joelho durante os diferentes tipos de agachamento. Todos os valores foram expressos através de médias e erro padrão.

TABELA 3

Análise de variância (ANOVA) - Atividade eletromiográfica (EMG) nas fases de aceleração (FA) e desaceleração (FD)

\begin{tabular}{lccccccc}
\hline $\begin{array}{c}\text { Músculos } \\
\text { (FA) }\end{array}$ & $\mathbf{F}$ & $\begin{array}{c}\text { Graus de } \\
\text { liberdade }\end{array}$ & $\mathbf{p}$ & $\begin{array}{c}\text { Músculos } \\
\text { (FD) }\end{array}$ & $\mathbf{F}$ & $\begin{array}{c}\text { Graus de } \\
\text { liberdade }\end{array}$ & $\mathbf{p}$ \\
VMO & 2,09 & $(4,28)$ & $<0,10$ & VMO & 1,33 & $(4,28)$ & $<0,28$ \\
RF & 1,24 & $(4,28)$ & $<0,31$ & RF & 0,61 & $(4,28)$ & $<0,65$ \\
VL & 2,18 & $(4,28)$ & $<0,09$ & VL & 1,41 & $(4,28)$ & $<0,25$ \\
BF & 1,11 & $(4,28)$ & $<0,37$ & BF & 0,14 & $(4,28)$ & $<0,96$ \\
GL & 1,28 & $(4,28)$ & $<0,30$ & GL & 1,19 & $(4,28)$ & $<0,33$ \\
TA & 4,59 & $(4,28)$ & $<0,50$ & TA & 1,61 & $(4,28)$ & $<0,20$ \\
\hline
\end{tabular}

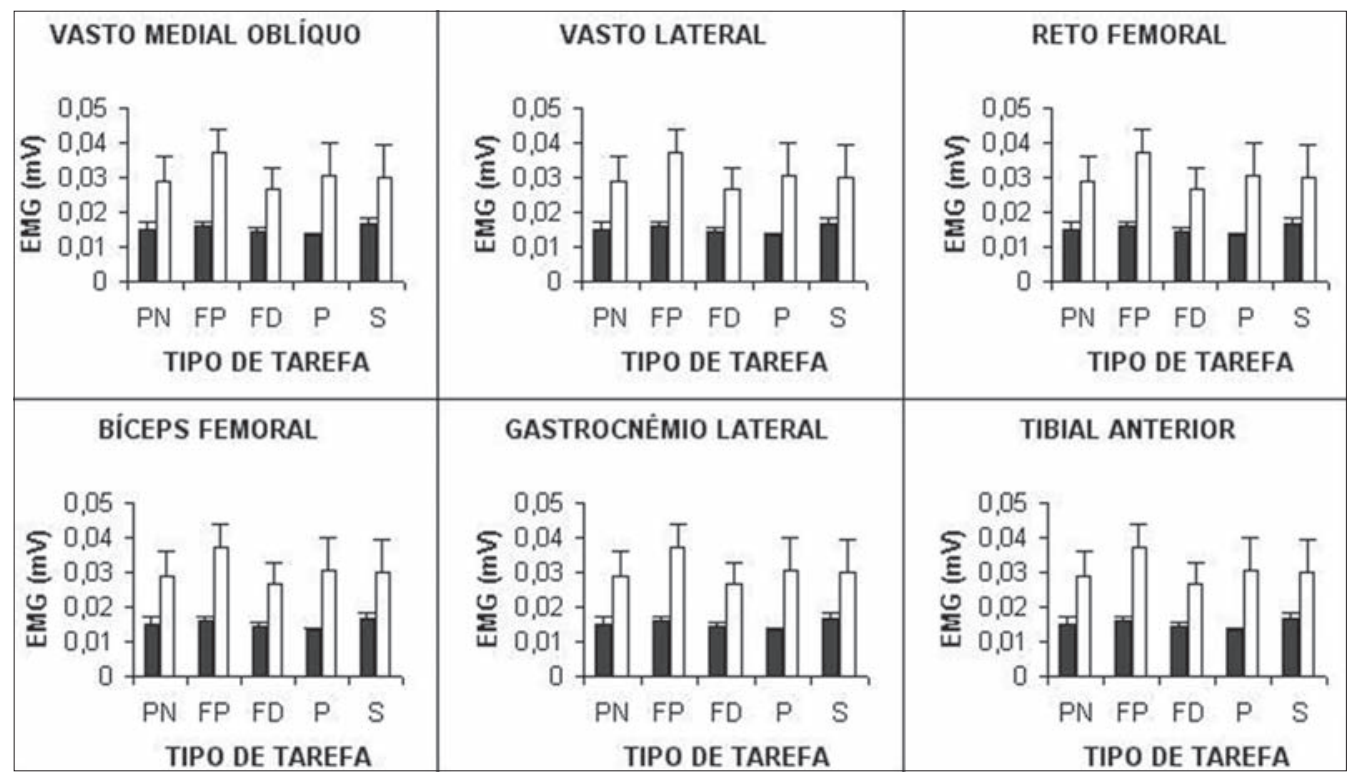

Figura 3 - Atividade eletromiográfica (EMG) dos músculos analisados durante os diferentes tipos de agachamento unipodal. As barras pretas representam a EMG na fase de aceleração e as barras brancas a EMG na fase de desaceleração do movimento. Todos os valores foram expressos através de médias e erro padrão. 


\section{DISCUSSÃO}

O desenho do estudo foi direcionado principalmente para prover informações relacionadas à influência do posicionamento do pé sobre a atividade eletromiográfica de músculos uniarticulares e biarticulares, durante o agachamento unipodal. $\mathrm{O}$ agachamento unipodal é uma tarefa de descarga de peso complexa, em que vários músculos atuam em conjunto(16). Além disso, o agachamento unipodal é uma tarefa empregada na reabilitação do sistema músculo-esquelético ${ }^{(13)}$ e está relacionada às atividades esportivas, sendo importante para o drible, salto e balanço do atleta( ${ }^{(15)}$. Mais recentemente tem sido sugerido que esta tarefa, se executada com o pé posicionado em declive de $25^{\circ}$, poderia favorecer a recuperação de sujeitos portadores de tendinite patelar ${ }^{(14)}$.

Apesar da relevância do agachamento unipodal nas atividades atléticas e de reabilitação, poucos trabalhos investigaram o comportamento eletromiográfico dos principais músculos envolvidos no agachamento unipodal. Hung e Gross ${ }^{(3)}$ investigaram a influência da inclinação medial e lateral do pé, durante o agachamento unipodal, sobre a atividade EMG. Eles não encontraram diferença na relação entre o vasto medial oblíquo e o vasto lateral, utilizando uma cunha de $10^{\circ}$ para inclinar o pé(3). Estes resultados são contrários aos resultados observados por Hertel et al.(6), que encontraram um aumento na atividade EMG do vasto medial e glúteo médio durante o agachamento com utilização de órteses, corrigindo o retropé no sentido médio-lateral. Estes autores ${ }^{(6)}$ não observaram correlação com o sentido da inclinação da órtese, ou seja, independentemente da correção postural, o emprego da órtese determinou aumento da atividade eletromiográfica do vasto medial e glúteo médio.

Nossos resultados não mostraram diferenças na atividade EMG durante as tarefas propostas, corroborando os resultados de Hung e Gross ${ }^{(3)}$. A diferença pode ser atribuída ao fato de que em nosso estudo utilizamos uma cunha de $10^{\circ}$ que causou alterações do posicionamento do pé como um todo, enquanto Hertel et al. (6) utilizaram uma órtese apenas no retropé (dentro do calçado), o que causou uma alteração local de uma região do pé. Além disso, diferenças na execução da tarefa, onde Hertel et al.(6) não controlaram a posição do tronco e a velocidade angular do joelho, poderiam ter influenciado os resultados.

\section{REFERÊNCIAS}

1. Ninos J C, Irrgang JJ , Burdett R, Weiss J R. Electromyographic analysis of the squat performed in self-selected lower extremity neutral rotation and 30 degrees of lower extremity turn-out from the self-selected neutral position. J Orthop Sports Phys Ther. 1997;25:307-15.

2. Anderson R, Courtney C, Carmeli E. EMG analysis of the vastus medialis/vastus lateralis muscles utilizing the unloaded narrow- and wide-stance squats. J Sport Rehabil. 1998;7:236-47.

3. Hung YJ , Gross MT. Effect of foot position on eletromyographic activity of the vastus medialis oblique and vastus lateralis during lower extremity weight-bearing activities. J Orthop Sports Phys Ther. 1999;29:93-102.

4. Escamilla RF, Flesig GS, Zheng N, Lander J E, Barrentine SW, Andrews J R, et al. Effects of technique variations on knee biomechanics during the squat and leg press. Med Sci Sports Exerc. 2001;33:1552-66.

5. Lam PL, Ng GYF. Activation of the quadriceps muscle during semisquatting with different hip and knee positions in patients with anterior knee pain. Am J Phys Med Rehabil. 2001;80:804-8.

6. Hertel J, Sloss BR, Earl J E. Effect of foot orthotics on quadriceps and gluteus medius electromyographic activity during selected exercises. Arch Phys Med Rehabil. 2005;86:26-30.

7. McCaw ST, Melrose DR. Stance width and bar load effects on leg muscle activity during the parallel squat. Med Sci Sports Exerc. 1999;31:428-36.

8. Escamilla RF. Knee biomechanics of the dynamic squat exercise. Med Sci Sports Exerc. 2001;33:127-41.

9. Richardson C, Bullock MI. Changes in muscle activity during fast, alternating flexion-extension movements of the knee. Scand J Rehabil Med. 1986;18:51-8.
Richardson e Bullock ${ }^{(9)}$ verificaram que o aumento da velocidade do movimento de flexão-extensão do joelho fez com que a atividade EMG do reto femoral e dos isquiotibiais aumentasse, enquanto a atividade EMG do vasto medial e do vasto lateral não sofreu alteração. Hagood et al.(10) encontram um grande aumento da atividade EMG antagonista do quadríceps e dos isquiotibiais empregando um aumento da velocidade articular do joelho. No nosso estudo monitoramos a velocidade angular do joelho nos cinco tipos de agachamento realizados, excluindo a influência da velocidade sobre a EMG.

Segundo Henry et al.(11), a atividade EMG dos músculos da coxa é influenciada por deslocamentos posturais no sentido lateral e ântero-posterior. Nesse mesmo sentido, outro estudo(12) verificou alterações EM G do músculo sóleo durante a realização de agachamentos sobre superfície instável; esses autores relacionaram as alterações EMG do sóleo ao seu papel no controle postural. Para diminuir o efeito do controle postural sobre a EMG, também efetuamos o controle do deslocamento do tronco.

No âmbito clínico, um protocolo empregando exercícios de agachamento unipodal associado ao posicionamento do pé sobre uma cunha com declive de $25^{\circ}$ foi capaz de causar melhora clínica em atletas com tendinite patelar ${ }^{(11)}$. Comparado com nosso estudo, este maior declive $\left(25^{\circ}\right)$ manteve o tornozelo em flexão plantar, o que poderia favorecer um diferente padrão de recrutamento muscular do quadríceps justificando a melhora do quadro inflamatório. Futuros estudos explorando o comportamento cinemático, cinético e eletromiográfico são necessários para melhor compreender os resultados positivos no tratamento da tendinite patelar.

\section{CONCLUSÃO}

Nossos resultados sugerem que alterações do posicionamento do pé durante o agachamento unipodal, de acordo com a metodologia descrita, não provocam alterações no padrão de recrutamento muscular dos principais músculos envolvidos na tarefa.

Todos os autores declararam não haver qualquer potencial conflito de interesses referente a este artigo.

10. Hagood S, Solomonow M, Baratta R, Zhou BH, D'Ambrosia R. The effect of joint velocity on the contribution of the antagonist musculature to knee stiffness and laxity. AmJ Sports Med. 1990;18:182-7.

11. Henry SM, Fung J , Horak FB. Control of stance during lateral and anterior/posterior surface translations. IEEE Trans Rehabil Eng. 1998;6:32-42.

12. Anderson K, Behm DG. Trunk muscle activity increases with unstable squat movements. Can J Appl Physiol. 2005;30:33-45.

13. Ng GY, Cheng J M. The effects of patellar taping on pain and neuromuscular performance in subjects with patellofemoral pain syndrome. Clin Rehabil. 2002; 16:821-7.

14. Young MA, Cook J L, Purdam CR, Kiss ZS, Alfredson H. Eccentric decline squat protocol offers superior results at 12 months compared with traditional eccentric protocol for patellar tendinopathy in volleyball players. $\mathrm{Br} J$ Sports Med. 2005;39:102-5.

15. Zeller BL, McCrory J L, Kibler WB, Tymothy UL. Differences in kinematics and electromyographic activity between men and women during single-legged squat. AmJ Sports Med. 2003;31:449-58.

16. Tagesson S, Öberg B, Kvist J . Passive and dynamic translation in the knee is not influenced by knee exercises in healthy individuals. Scand J Med Sci Sports. 2005;15:139-47.

17. Bevilaqua-Grossi D, Felicio LR, Simões R, et al. Avaliação eletromiográfica dos músculos estabilizadores da patela durante exercício isométrico de agachamento em indivíduos com síndrome da dor femoropatelar. Rev Bras Med Esporte. 2005;11:159-63.

18. Basmajian J V, Blumenstein. Electrode placement in EMG biofeedback. Baltimore: Williams and Wilkins, 1980;79-86. 